Biochemical Journal - Accelerated Publication

Revised manuscript, Paper number: BJ2015/1089

\title{
The STAS domain of mammalian SLC26A5 prestin harbors an anion-binding site
}

Graziano Lolli ${ }^{* 1,2}$, Elisa Pasqualetto ${ }^{* 1}$, Elisa Costanzi ${ }^{*}$, Greta Bonetto ${ }^{*} \&$ Roberto Battistutta ${ }^{* 2}$

*Department of Chemical Sciences, University of Padua, via Marzolo 1, 35131 Padua, Italy.

${ }^{1}$ These authors contributed equally to this work.

${ }^{2}$ Corresponding authors. Department of Chemical Sciences, via Marzolo 1, 35131 Padua ITALY.

Tel.+39.049.827.5262, Fax:+39.049.827.5829, e-mails graziano.lolli@unipd.it, roberto.battistutta@unipd.it. 


\begin{abstract}
Prestin is a unique ATP- and $\mathrm{Ca}^{2+}$-independent molecular motor with piezoelectric characteristic responsible for the electromotile properties of mammalian cochlear outer hair cells, that is the capacity of these cells to modify their length in response to electric stimuli. This "electromotility" is at the basis of the exceptional sensitivity and frequency selectivity distinctive of mammals. Prestin belongs to the SLC26 family of anion transporters and needs anions to function properly, in particular chloride. By X-ray crystallography here we reveal that the STAS domain of mammalian prestin, considered an "incomplete" transporter, harbor an unanticipated anion-binding site. In parallel we present the first crystal structure of a prestin STAS domain from a non-mammalian vertebrate prestin (chicken) that behaves as a "full" transporter. Notably, in chicken STAS the anion-binding site is lacking due to a local structural rearrangement, indicating that the presence of the STAS anion-binding site is exclusive to mammalian prestin.
\end{abstract}

\title{
SUMMARY STATEMENT
}

The STAS domain of mammalian prestin harbors an anion-binding site absent in non-mammalian homologues. This is correlated to different prestin functions, full anion transport in non-mammals and incomplete transport coupled to electromotility and a mechanically amplified hearing process in mammals.

\section{SHORT TITLE}

Mammalian prestin STAS holds an anion-binding site

\section{KEYWORDS}

Anion transporters; electromotility; prestin; SLC26; STAS domain; crystal structure 


\section{INTRODUCTION}

In order to get insights into the anion-binding properties of prestin, a member of the SLC26 family of anion transporters, we determined crystal structures of a mammalian STAS domain in presence of several monovalent anions known to modulate activity in cell and the first crystal structure of a domain from a non-mammalian vertebrate homologue. While in non-mammals prestin has been shown to be an electrogenic anion transporter [1], in mammals prestin is considered an incomplete transporter [2]. Mammalian prestin function relies on the presence of monovalent anions such as $\mathrm{Cl}^{-}$ , $\mathrm{Br}^{-}, \mathrm{I}^{-}, \mathrm{HCO}_{3}^{-}, \mathrm{NO}_{3}^{-}$[2] and $\mathrm{SCN}^{-}$[3]. Physiologically the most relevant is chloride that has been proposed to be the extrinsic voltage sensor [2]. The incomplete transport of chloride in mammals is considered at the basis of the ATP- and $\mathrm{Ca}^{2+}$-independent motor properties with piezoelectric characteristic of prestin, giving rise to cochlear outer-hair cells (OHCs) electromotility [4].

Topologically, prestin is divided into a large transmembrane (TM) domain and a cytosolic C-term portion composed by the STAS (Sulphate Transporters and Anti-Sigma factor antagonist) domain [5]. The 3D structure of STAS domains from rat [6], E. coli [7] and M. tuberculosis [8] show a conserved folding core, constituted by a central $\beta$-sheet composed of 5-6 $\beta$-strands, surrounded by 5 $\alpha$-helices. Recently, the experimentally validated 3D model of the mammalian TM domain of prestin identified a central anion-binding site in the middle of the domain, along as a possible pathway leading anions from the cytosol [9]. The general architecture of this model has been confirmed by the very recent crystal structure of a prokaryotic SLC26 fumarate transporter from the bacterium Deinococcus geothermalis [10]. However, the cytosolic recruitment site for anions has not been identified yet.

\section{MATERIALS AND METHODS}

\section{Cloning of chicken STAS}

The STAS domain from Gallus gallus, deleted of the variable loop, was cloned with a protocol similar to that used for the Rattus norvegicus domain, previously described [6]. Briefly, the nucleotide sequences corresponding to amino acids [511-569] and [652-733] of Gallus gallus prestin were separately amplified by PCR and ligated with a GlySer insert into the pBluescript II SK (+/-) storage phagemid (Agilent Technologies). The final construct S[511-569]GS[652-733] was inserted into the pET SUMO vector (Invitrogen).

\section{Proteins production and purification}

STAS domains from Rattus norvegicus (rPres STAS), sequence [505-563]GS[637-718], and Gallus gallus (cPres STAS) were produced according to the protocol previously described [6]. Briefly, the SUMO fusion proteins were produced in E. coli BL21(DE3) grown at $37^{\circ} \mathrm{C}$ and induced for $5 \mathrm{~h}$ at $30{ }^{\circ} \mathrm{C}$. Bacteria were suspended in $50 \mathrm{mM} \mathrm{Na}_{2} \mathrm{HPO}_{4}, 300 \mathrm{mM} \mathrm{NaCl}, 10 \mathrm{mM}$ imidazole, $10 \mathrm{mM} \beta$ mercaptoethanol, $\mathrm{pH} 8.0$ (buffer A), lysed and applied onto a His-Trap affinity column (GE Healthcare) equilibrated with buffer $\mathrm{A}$. The fusion proteins were eluted at $150 \mathrm{mM}$ imidazole and incubated with the SUMO protease 1 (LifeSensors) overnight at $4{ }^{\circ} \mathrm{C}$. The (His) 6 -SUMO-tag and the protease were removed through a His-Trap column. The final proteins were further purified by means of a Superdex 75 prep-grade 16/60 (GE Healthcare) SEC column equilibrated with $20 \mathrm{mM}$ Tris- $\mathrm{HCl}, 150 \mathrm{mM} \mathrm{NaCl}, 5 \mathrm{mM}$ DTT, $\mathrm{pH}$ 7.5. Pooled samples were concentrated at around 15 and $35 \mathrm{mg} / \mathrm{ml}$ for rPres and cPres STAS, respectively, and flash-frozen in liquid nitrogen.

\section{Crystallization, structure determination, refinement and final models analysis}

All crystals of rPres STAS in complex with anions were prepared with the vapour diffusion technique, using 1.8-2.0 M Ammonium sulfate, 5\% (v/v) PEG400, 0.1 M MES pH 6.5 and 0.1\% octyl- $\beta$-D-glucopyranoside as precipitant solution, at $293 \mathrm{~K}$. In particular, complexes with bromide, 
thiocyanate and nitrate were prepared by cocrystallization with $125 \mathrm{mM} \mathrm{KBr}, 250 \mathrm{mM} \mathrm{NaSCN}$ and $100 \mathrm{mM} \mathrm{NaNO}_{3}$, respectively, complexes with chloride and iodide by soaking with $1 \mathrm{M} \mathrm{NH}_{4} \mathrm{Cl}$ and $500 \mathrm{mM} \mathrm{KI}$, respectively. Attempts to identify chloride bound to STAS failed in the case of soakings with less than $1 \mathrm{M}$ chloride, indicating that the chloride seen in the anion-binding site is not carried throughout the purification process. Even with $1 \mathrm{M} \mathrm{NH} \mathrm{N}_{4} \mathrm{Cl}$ the anomalous signal at $2 \AA$ was much weaker than that observed for crystals soaked with $500 \mathrm{mM} \mathrm{KI}$ collected at the same wavelength, strongly indicating that all four anomalous maxima identified in the structure soaked with $0.5 \mathrm{M} \mathrm{KI}$ must be assigned to iodide and not to chloride.

Crystals of cPres STAS were prepared by vapour diffusion using $1.5 \mathrm{M}$ ammonium sulfate, $12 \%$ (v/v) glycerol, $0.1 \mathrm{M}$ Tris $\mathrm{pH} 8.5$ and $300 \mathrm{mM}$ ammonium oxalate as precipitant solution. Diffraction data were collected on cryoprotected crystals $(20 \%$ glycerol) at $100 \mathrm{~K}$, at the XDR1 beamline of the Elettra Synchrotron (Trieste), at $2 \AA$ wavelength for rPres STAS in complex with chloride, iodide and thiocyanate, at $0.915 \AA$ for rPres STAS in complex with bromide and for cPres STAS and at $1.2 \AA$ for rPres STAS in complex with nitrate. Data were processed with XDS [11] and Aimless [12], structures were solved by molecular replacement with Phaser [13] using the structure of the apo rPres STAS (PDB 3LLO) as search model. cPres chains were initially reconstructed by automatic procedure using the "Model building" tool implemented in Phenix [14]. Initial models were refined alternating cycles of automatic refinement with phenix.refine [14] and manual model building with COOT [15]. Statistics on data collection and final models are reported in Table I. Ramachandran statistics (\% Favoured/Allowed/Outliers): STAS-Cl = 100/0/0; STAS-Br $=$ 99/1/0, STAS-I = 99/1/0, STAS-SCN = 99/1/0, STAS-nitrate $=99 / 1 / 0$, chickenSTAS = 96/4/0. Electrostatic calculations were performed solving the Poisson-Boltzmann equation by the APBS tool [16], with atomic charges and radius information provided by PDB2PQR [17] on the web service http://www.poissonboltzmann.org/. Analysis of molecular interfaces for the cPres STAS domain were performed by PISA [18].

\section{RESULTS AND DISCUSSION}

\section{Anion-binding site in mammalian prestin STAS}

The five crystal structures of mammalian STAS (from rat prestin, rPres) presented here reveal for the first time that $\mathrm{Cl}^{-}, \mathrm{Br}^{-}, \mathrm{I}^{-}, \mathrm{SCN}^{-}$and $\mathrm{NO}_{3}{ }^{-}$effectively bind in the same place of the domain, concurring in the identification of an unanticipated unique anion-binding site. The presence and location of $\mathrm{Cl}^{-}, \mathrm{Br}^{-}, \mathrm{I}^{-}$and $\mathrm{SCN}^{-}$were unambiguously determined by means of the proper anomalous signal (Supplementary Figure S1). Statistics on X-ray diffraction data and final models are reported in Table 1.

The overall 3D structure of rPres STAS is poorly affected by the binding of anions, all located in the same cleft between loops $\beta 2-\alpha 1$ and $\beta 3-\alpha 2$ (Figure 1). The anion-binding site is a relatively large and deep cavity, situated in a region proposed to facing the TM domain [6]. This localization suggests that effectively it can be the very first binding site for intracellular chloride, that is then translocated to the anion pathway identified in the TM domain [9]. The cavity has a positive potential, mainly due to the dipoles of backbone NHs from the N-termini of helices $\alpha 1$ and $\alpha 2$ (Figure 2). These NHs establish ion-dipole interactions with $\mathrm{Cl}^{-}, \mathrm{Br}^{-}$and $\mathrm{I}^{-}$(Supplementary Figure S2A,B,C). Similar ion-dipole interactions between chloride and backbone NHs from the N-termini of alpha-helices are found in the $\mathrm{ClC}$ family of chloride channels and transporters [19]. Another correspondence between STAS and $\mathrm{ClC}$ is the presence of an acidic residue near the bound anion, D653 in STAS (Figure 1) and E148 in ClC, where it is considered the gate of the selectivity filter. By analogy, one can hypothesizes that the conserved D653 plays a functional role in prestin.

Bound in similar position, nitrate and thiocyanate make hydrogen bonds with I544 and D653 NHs (Supplementary Figure S2D,E). Notably, we could not detect any binding for anions that are unable to induce NLC such as sulphate [2] and oxalate [1,20], nor for acetate, that can induce NLC but 
with a significant decrease in the slope factor [2]. This indicates that the STAS anion-binding site is specific for those anions that are able to fully modulate mammalian prestin function.

The cavity remains unchanged upon anion binding (Figure 1,2) indicating that the binding site is preformed, ready to accommodate and release anions without the need of conformational rearrangements. These observations are in accordance with the low affinity (low millimolar) shown by anions as determined on full-length prestin in cochlear $\mathrm{OHC}$ [2], and seems a necessary prerequisite for the fast rate at which prestin must operate, in the range of several tens of $\mathrm{kHz}$.

Based on the anomalous signal, a second chloride and three other iodide ions were identified bound on the surface of the STAS domain, in positions where neither $\mathrm{Br}^{-}$nor $\mathrm{SCN}^{-}$or $\mathrm{NO}_{3}{ }^{-}$were found. The solvent exposed location of the second chloride (not in a positively charged deep cleft like the anion-binding site), the lack of strong interactions with STAS and the fact that only iodide is found in the same position suggest that this is an unspecific binding. However, we cannot completely rule out that, even with a lower affinity, this site might have some significance for the interaction of the domain with the physiological relevant chloride.

\section{Crystal structure of a non-mammalian prestin STAS}

We also determined the crystal structure of the STAS domain from chicken prestin (cPres), the first one from a non-mammalian vertebrate (Supplementary Figure S3A,B). The overall architecture resembles that of the rat homologue, with similar arrangements of secondary structure elements (Figure 3A). In each molecule three cysteins cluster to form a binding site for ions, most probably $\mathrm{Zn}^{2+}$ in our structure on the basis of $\mathrm{x}$-ray fluorescence measurement on the crystal and geometry of coordination (Supplementary Figure S3C). The physiological relevant metal ion and its role is still unclear. These cysteines are conserved in avian prestins and in a species of turtle, the chinese softshell turtle Pelodiscus sinensis (Supplementary Figure S3D), suggesting a specific role in these species.

The major structural difference between chicken and rat STAS domains is at the level of the $\beta 2-\alpha 1$ loop and the following $\alpha 1$ helix (Figure 3B). The $\beta 2-\alpha 1$ loop is closer to the $\beta 3-\alpha 2$ loop (whose position is conversely very similar in chicken and rat), with the consequence that the anion-binding site is absent in chicken STAS (Figure 3C,D) indicating that anion-binding is a property peculiar to the mammalian domain. The interaction between loops $\beta 2-\alpha 1$ and $\beta 3-\alpha 2$ in chicken is stabilized by the formation of a short parallel $\beta$-sheet (Figure 3B,D). Interestingly, the sequence around loop $\beta 2$ $\alpha 1$ is fully conserved in mammals (INAPI), while is different and variable in non-mammals (Figure 3E) [21], indicating a close relationship between the evolutionary appearance and maintenance of this sequence and the correlated anion-binding and electromotile properties in prestin.

\section{Role of the STAS domain in mammalian prestin}

It is known that the presence of a correctly folded STAS domain is essential for prestin function [5]. Results obtained on prestin chimeras where mammalian and non mammalian STAS domains have been exchanged [20] show that the original functional properties (NLC or transport) are maintained, at least at the course-grained level accessible to the electro-physiological measurements. Our results clarify that this is due to the conservation of the fold between mammalian and non-mammalian STAS (rmsd between rat and chicken STAS $1.2 \AA$, with $60 \%$ sequence identity). Further, while the TM domain is the main responsible for NLC/electromotility in mammals and transport in nonmammals, the STAS domain is responsible for the fine tuning of these properties. We propose that the anion-binding site in the STAS domain of mammalian prestins represents a reservoir of readyto-use anions, increasing their local concentration, for the fast rate at which this molecular motor must operate, up to $120 \mathrm{kHz}$ in the ultrasonic hearing of some cetaceans [22], values never reached by electro-physiological measurements on electromotile prestin. This rapidness is not necessary for transport in non-mammalian prestin for which the presence of a STAS anion-binding site does not seem essential. The resting level of chloride in OHCs is near or below $10 \mathrm{mM}$ [23] and in-side-out patches measurements from rat OHCs have shown that the half-activating chloride concentration is 
around $6 \mathrm{mM}$ for full-length prestin [2]. This suggests that in physiological conditions half or more of the STAS anion-binding sites are occupied by chloride and considering that prestin acts as dimer or tetramer $[24,25]$, that all functional oligomers have at least one anion permanently bound.

Results presented here reveal for the first time important structural differences between mammalian (incomplete transporters) and non-mammalian (full-antiporters) prestins, directly correlated to their different function. Very recently, the first crystal structure of a bacterial prestin homologue, the SLC26 prokaryotic fumarate transporter from Deinococcus geothermalis, has been published [10]. This structure shows that the TM domain of SLC26 transporters is composed by 14 transmembrane segments organized in two inverted repeats, confirming our previous $3 \mathrm{D}$ model of the mammalian transmembrane domain of prestin [9]. However it does not present indications on the localization of possible anion-binding site in prestin and on the relative orientation of the transmembrane and STAS domains. Our findings complement such structural information resulting in a better picture of the structural and functional organization of SLC26 transporters. Here we show that the STAS domain of mammalian prestin, unlike the non-mammalian one, harbors a unique anion-binding site important for the fine regulation of the high-frequency electromotile properties of this exceptional molecular motor. We propose that the STAS domain structurally interacts with the TM domain (deletion of STAS abrogate function [5]), and that it is oriented with the anion-binding site facing the TM domain, for a fast anion translocation. 


\section{Acknowledgments}

Authors thank the staff at ELETTRA beamline XDR1 for on-site assistance.

\section{Funding}

The research leading to these results has received funding from the European Community's Seventh Framework Programme (FP7/2007-2013) under BioStruct-X (grant agreement $N^{\circ} 283570$ ). This work was supported by a grant from Fondazione Cassa di Risparmio di Padova e Rovigo to R. B. ("Progetto di Eccellenza").

\section{Author contributions}

G.L., E.P. and R.B. designed the study and analyzed data; G.L., E.P., E.C. and G.B. performed the experiments; R.B. wrote the paper.

\section{Conflict of interest}

The authors declare that they have no conflict of interest.

\section{Accession codes}

Coordinates and structure factors have been deposited in the Protein Data Bank under accession code 5EUU (ratSTAS/ $/ \mathrm{Cl}^{-}$), 5EUS (ratSTAS/Br'), 5EUZ (ratSTAS/I'), 5EUW (rat STAS/nitrate), 5EUX (rat STAS/thiocyanate) and 5EZB (chicken STAS). 


\section{REFERENCES}

1. Schaechinger TJ, Oliver D. (2007) Nonmammalian orthologs of prestin (SLC26A5) are electrogenic divalent/chloride anion exchangers. Proc Natl Acad Sci U S A 104, 7693-7698.

2. Oliver D, He DZ, Klocker N, Ludwig J, Schulte U, Waldegger S, Ruppersberg JP, Dallos P, Fakler B. (2001) Intracellular anions as the voltage sensor of prestin, the outer hair cell motor protein. Science 292, 2340-2343.

3. Schanzler M, Fahlke C. (2012) Anion transport by the cochlear motor protein prestin. J Physiol 590, 259-272.

4. Dallos P, Fakler B. 2002. Prestin, a new type of motor protein. Nature reviews 3, 104-111.

5. Zheng J, Du GG, Matsuda K, Orem A, Aguinaga S, Deak L, Navarrete E, Madison LD, Dallos P. (2005) The C-terminus of prestin influences nonlinear capacitance and plasma membrane targeting. Journal of cell science 118, 2987-2996.

6. Pasqualetto E, Aiello R, Gesiot L, Bonetto G, Bellanda M, Battistutta R. (2010) Structure of the cytosolic portion of the motor protein prestin and functional role of the STAS domain in SLC26/SulP anion transporters. J Mol Biol 400, 448-462.

7. Babu M, Greenblatt JF, Emili A, Strynadka NC, Reithmeier RA, Moraes TF. (2010) Structure of a SLC26 anion transporter STAS domain in complex with acyl carrier protein: implications for E. coli YchM in fatty acid metabolism. Structure 18, 1450-1462.

8. Sharma AK, Ye L, Baer CE, Shanmugasundaram K, Alber T, Alper SL, Rigby AC. (2011) Solution structure of the guanine nucleotide-binding STAS domain of SLC26-related SulP protein Rv1739c from Mycobacterium tuberculosis. J Biol Chem 286, 8534-8544.

9. Gorbunov D, Sturlese M, Nies F, Kluge M, Bellanda M, Battistutta R, Oliver D. (2014) Molecular architecture and the structural basis for anion interaction in prestin and SLC26 transporters. Nat Commun 5, 3622.

10. Geertsma ER, Chang YN, Shaik FR, Neldner Y, Pardon E, Steyaert J, Dutzler R. (2015) Structure of a prokaryotic fumarate transporter reveals the architecture of the SLC26 family. Nat Struct Mol Biol. 22, 803-808.

11. Kabsch W. (2010) XDS. Acta Crystallogr D Biol Crystallogr 66, 125-132.

12. Evans PR, Murshudov GN. (2013) How good are my data and what is the resolution? Acta Crystallogr D Biol Crystallogr 69, 1204-1214.

13. McCoy AJ, Grosse-Kunstleve RW, Adams PD, Winn MD, Storoni LC, Read RJ. (2007) Phaser crystallographic software. J Appl Crystallogr 40, 658-674.

14. Adams PD, Afonine PV, Bunkoczi G, Chen VB, Davis IW, Echols N, Headd JJ, Hung LW, Kapral GJ, Grosse-Kunstleve RW, McCoy AJ, Moriarty NW, Oeffner R, Read RJ, Richardson DC, Richardson JS, Terwilliger TC, Zwart PH. (2010) PHENIX: a comprehensive Python-based system for macromolecular structure solution. Acta Crystallogr D Biol Crystallogr 66, 213-221.

15. Emsley P, Lohkamp B, Scott WG, Cowtan K. (2010) Features and development of Coot. Acta Crystallogr D Biol Crystallogr 66, 486-501.

16. Baker NA, Sept D, Joseph S, Holst MJ, McCammon JA. (2001) Electrostatics of nanosystems: application to microtubules and the ribosome. Proceedings of the National Academy of Sciences of the United States of America 98, 10037-10041.

17. Dolinsky TJ, Czodrowski P, Li H, Nielsen JE, Jensen JH, Klebe G, Baker NA. (2007) PDB2PQR: expanding and upgrading automated preparation of biomolecular structures for molecular simulations. Nucleic Acids Res 35, W522-525.

18. Krissinel E, Henrick K. (2007) Inference of macromolecular assemblies from crystalline state. J Mol Biol 372, 774-797.

19. Dutzler R. 2006. The ClC family of chloride channels and transporters. Curr Opin Struct Biol 16:439-446.

20. Schaechinger TJ, Gorbunov D, Halaszovich CR, Moser T, Kugler S, Fakler B, Oliver D. 2011. A synthetic prestin reveals protein domains and molecular operation of outer hair cell 
piezoelectricity. EMBO J 30:2793-2804.

21. Liu Z, Li GH, Huang JF, Murphy RW, Shi P. 2012. Hearing aid for vertebrates via multiple episodic adaptive events on prestin genes. Mol Biol Evol 29:2187-2198.

22. Liu Y, Rossiter SJ, Han X, Cotton JA, Zhang S. 2010. Cetaceans on a molecular fast track to ultrasonic hearing. Curr Biol 20:1834-1839.

23. Santos-Sacchi J, Song L, Zheng J, Nuttall AL. 2006. Control of mammalian cochlear amplification by chloride anions. J Neurosci 26:3992-3998.

24. Detro-Dassen S, Schanzler M, Lauks H, Martin I, zu Berstenhorst SM, Nothmann D, TorresSalazar D, Hidalgo P, Schmalzing G, Fahlke C. 2008. Conserved dimeric subunit stoichiometry of SLC26 multifunctional anion exchangers. J Biol Chem 283:4177-4188.

25. Zheng J, Du GG, Anderson CT, Keller JP, Orem A, Dallos P, Cheatham M. 2006. Analysis of the oligomeric structure of the motor protein prestin. J Biol Chem 281:19916-19924. 


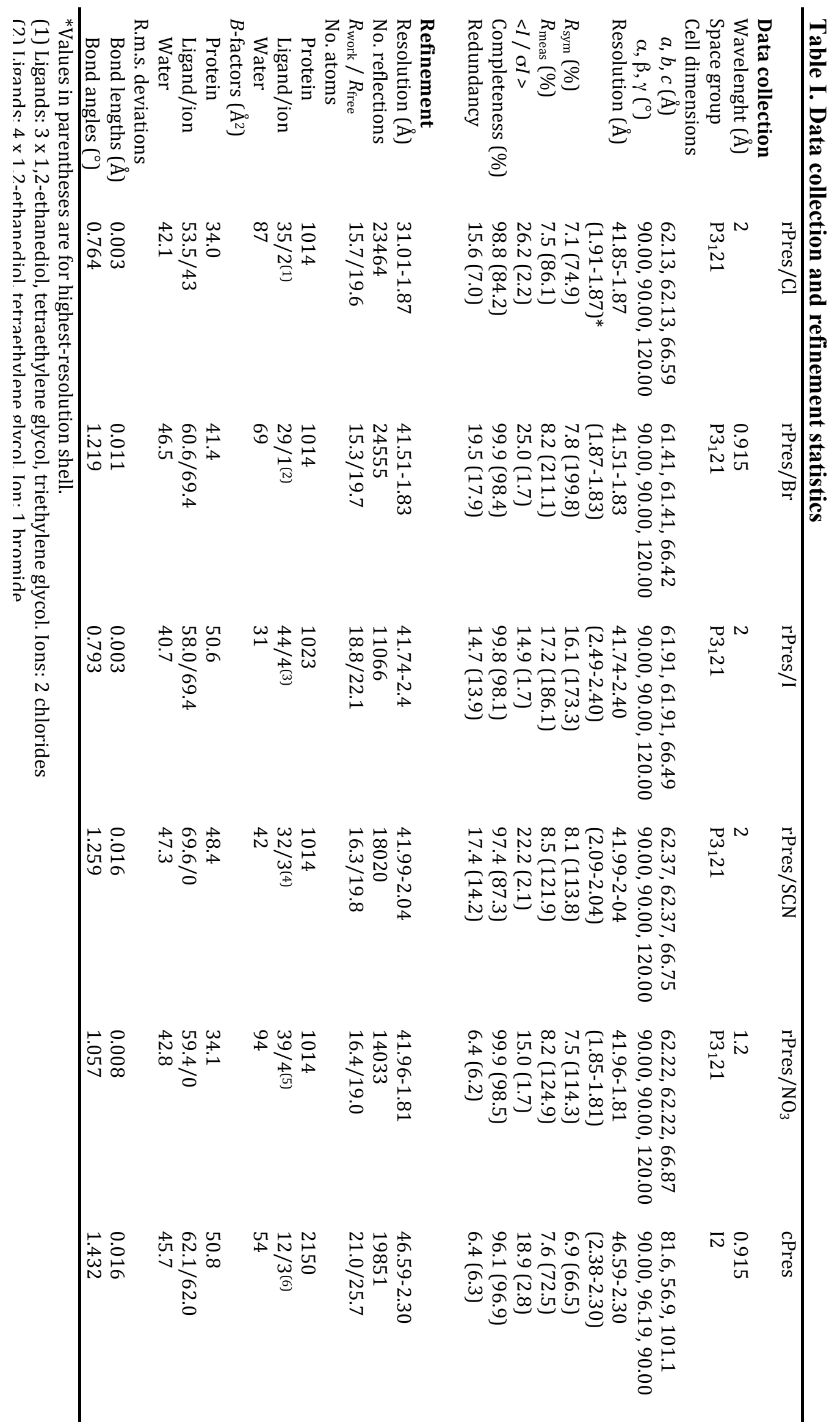



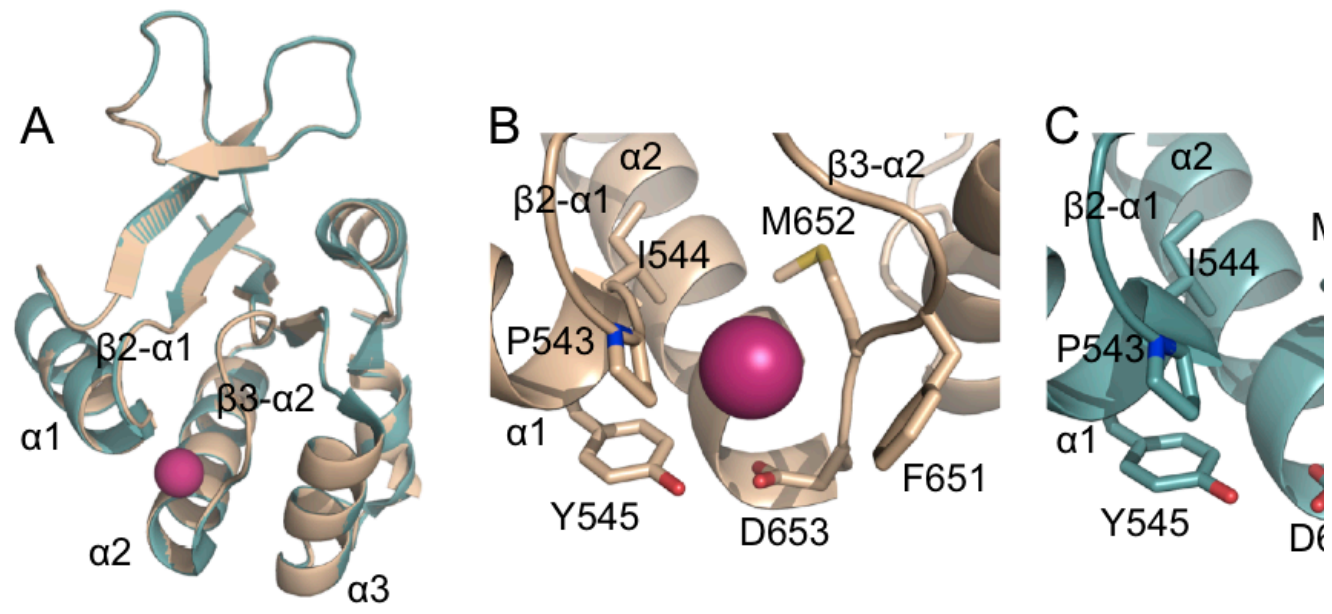

Figure 1. The anion-binding site in rPres STAS

(A) Superposition of rPres STAS in presence (wheat) and absence (cyan) of (magenta). Secondary structure elements and the overall 3D architecture of conserved. Close-up view of the anion-binding site in presence (B) and absence showing that binding does not alter the local structure. Helices $\alpha 1, \alpha 2$ and $\alpha 3$, loop $\alpha 2$ and main residues are indicated (see also Figure S1). 

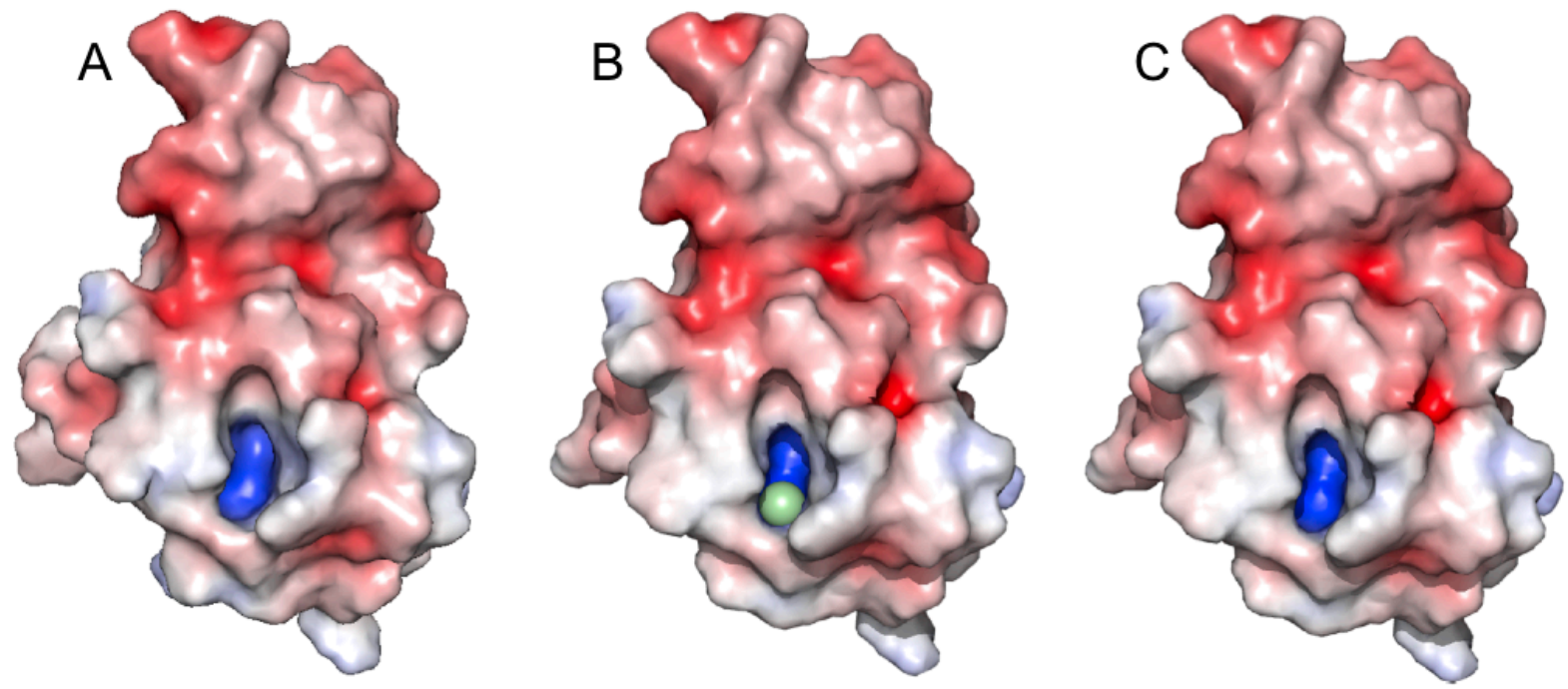

Figure 2. Molecular surface of rPres STAS colored according to electrostatics

In blue positive and in red negative potentials ( $\pm 5 \mathrm{kT} / \mathrm{e})$. (A) apo structure, PDB entry 3LLO [6], (B) with bound chloride (green sphere), (C) as in (B) without the anion. The binding site is a deep cavity with a positive potential that is not altered by anion binding (see also Figure S2). 
A
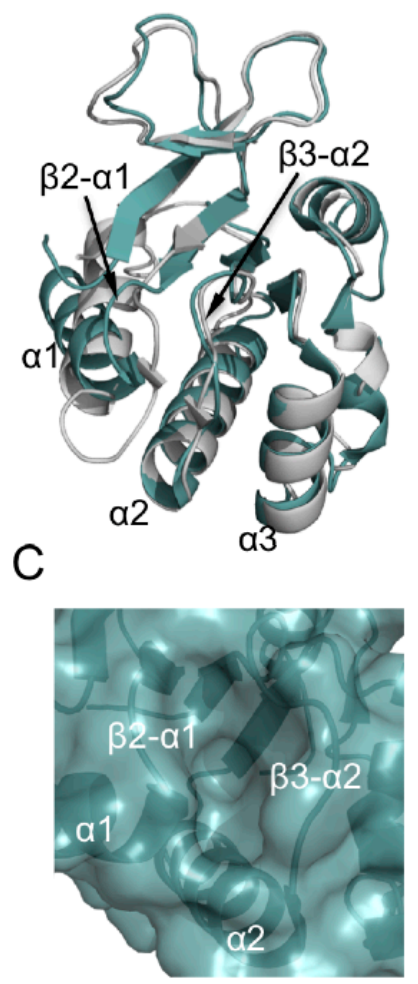

B
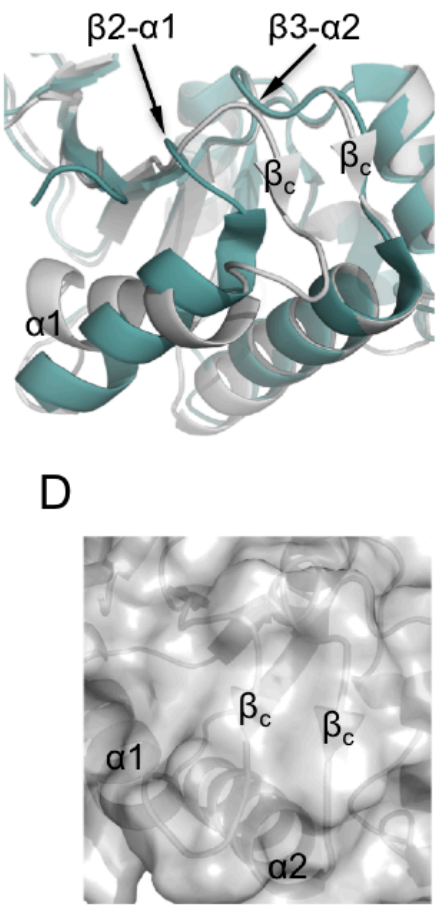

E

$\beta 2$

LLEEEEEELLLHHHHHHHHH

PGIKIFQINAPIYYANSDLY

PGIKIFQINAP IYYANSDLY

PGIKIFQINAP IYYANSDLY

PGIKIFQINAPIYYANSDLY

PGIKIFQINAPIYYANSDLY

PGIKIFQINAP IYYANSDLY

PGIKIFQINAP IYYANSDLY

PGIKIFQINAPIYYANSDLY

PGIKIFQINAPIYYANSDLY

PGIKIFQINAPIYYANSDLY

PGIKIFQINAPIYYANSDLY

AGIKIFQINAPIYYANSDLY

PGIKIFQINAP IYYANSDLY

$\beta 2 \quad \beta_{c} \quad \alpha 1$

LLEEEEELLLEELLLLHHHH PGIKIFQANTSLYFANSESY

PGIKIFQANTSLYFANSESY

PGIKIFQANTSLYFANSESY

Turkey

Finch

Anole lizard PGIKIFQANASLYFANSELY

Zebrafish

Stickleback

Fugu fish
SGIKIFQSNSSIYFANSELY

EGIKIFYSNASVYFVNSDLY

PGIFIFRSSATLYYANAEMY

\section{Figure 3. Comparison of rPres and cPres STAS domains}

(A) Superposition of rat (cyan) and chicken (grey) STAS shows that the secondary structure elements and the overall 3D architecture are generally conserved. The most relevant differences are the orientation of helix $\alpha 1$ and the position of loop $\beta 2-\alpha 1$ that in chicken interacts with loop $\beta 3-\alpha 2$ with the formation of a short $\beta$-sheet $\left(\beta_{\mathrm{C}}\right)(\mathbf{B})$. The consequence is that the rPres anion-binding site (C) is absent in cPres (D). (E) Alignment of residues around the $\beta 2-\alpha 1$ segment showing conservation of the INAPI stretch in mammals and sequence variation in non-mammals (adapted from [22]). Secondary structures $(\mathrm{L}=$ loop, $\mathrm{E}=\beta$-strand, $\mathrm{H}=$ helix) are indicated (see also Figure $\mathrm{S} 3)$. 